\title{
Research on Kinematics and Pouring Law of a Mobile Heavy Load Pouring Robot
}

\author{
Long Li $\mathbb{D}^{1,2}$ Chengjun Wang $\mathbb{D}^{1},{ }^{2}$ and Hongtao Wu ${ }^{1}$ \\ ${ }^{1}$ College of Mechanical and Electrical Engineering, Nanjing University of Aeronautics and Astronautics, Nanjing, China \\ ${ }^{2}$ College of Mechanical Engineering, Anhui University of Science and Technology, Huainan, China \\ Correspondence should be addressed to Chengjun Wang; 67155946@qq.com
}

Received 13 December 2017; Revised 7 March 2018; Accepted 3 April 2018; Published 23 May 2018

Academic Editor: Aki Mikkola

Copyright (c) 2018 Long Li et al. This is an open access article distributed under the Creative Commons Attribution License, which permits unrestricted use, distribution, and reproduction in any medium, provided the original work is properly cited.

\begin{abstract}
In order to meet the requirement of continuous pouring in many varieties and small batches in casting production, a mobile heavy load pouring robot is developed based on a new 4-UPU parallel mechanism due to its strong carrying capacity. Firstly, the instantaneous motion characteristics of the novel 4-UPU parallel mechanism with four degrees of freedom (DOF) are analyzed using screw theory. By using the geometric method, both the forward and inverse kinematic solutions of the proposed robot system are given out. Secondly, based on a common pouring ladle, the volume change of pouring liquid in pouring process and the relationship between tilting angular velocity and flow rate are analyzed, and the results show that the shape of the ladle and the design of the pouring mouth have great influence on the tilting model. It is an important basis for the division of the sectional model. Finally, a numerical example is given to verify the effectiveness of the developed tilting model. The mapping relation between the tilting model and the parallel mechanism shows that the pouring flow can be adjusted by controlling the movement of parallel manipulator. The research of this paper provides an important theoretical basis for the position control of mobile heavy load pouring robot and the research of pouring speed control.
\end{abstract}

\section{Introduction}

Pouring is the process of pouring the molten metal with high temperature from the ladle into the mould cavity. The adverse operating environment, such as dust, vibration, and noise, causes potential adverse effects on the personal security of workers. In order to solve this problem, various types of automatic pouring equipment have been developed for the pouring process $[1,2]$.

There are several commonly used pouring methods, such as bottom gating type, tilting type, and air pressure type [3]. Among them, the tilting pouring equipment is the most widely used because of its simple principle and convenient control. Back in the 1908, Benjamin invented the earliest hand cranked pouring machine [4]. By the second half of the 20th century, the United States, Japan, China, and other countries have developed various types of semiautomatic and fully automatic pouring machines [5-9]. At present, the structure of the title-typed automatic pouring equipment is still not changed greatly, and the movement mode is in accordance with the given walking route and tilting mode, which is suitable for mass production. However, in the face of more varieties and small quantities of castings, the production demand is increasing day by day. It is urgent to have automatic pouring equipment with movability, high flexibility, and high carrying capacity.

The parallel mechanism has the advantages of compact structure, large rigidity, strong bearing capacity, and high accuracy [10]. Thus, it is suitable for the tilting-typed automatic pouring equipment of actuators. HEXEL, Delta, Tricept, and many other parallel mechanisms have been extensively used in machine tools, steel, medical, aviation, and other fields [11]. For the flow control of tilting pouring equipment, early automatic pouring system was finished by preteaching. However, this method has many problems in inappropriate speed and pouring, for example, the teaching process needs a lot of time and manpower and the accuracy is not high [12]. In order to solve these problems, a lot of pouring flow control methods have been proposed. Noda et al. put forward a complete set of flow control and position control 


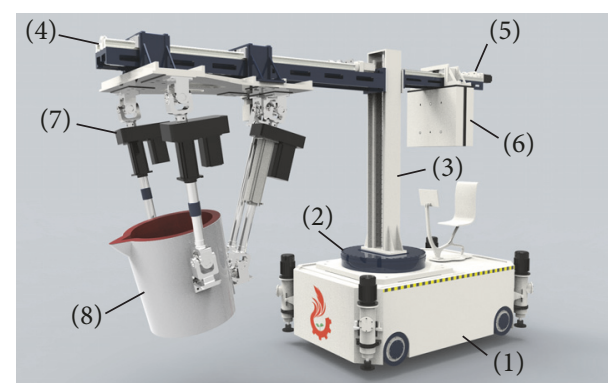

FIGURE 1: Hybrid truss type movable pouring robot. (1) 4WD mobile platform. (2) Slewing device. (3) Hoisting device. (4) Forward device. (5) Backward device. (6) Counterweight device. (7) Parallel manipulator. (8) End-effector.

method based on the liquid metal flow model. The molten steel sputtering mechanism and vibration suppression were studied, which achieved a good effect in practice [13-16]. Sun et al. discretized the fluid and simulated the tilting process, revealing the general law of fluid dumping [17]. Pan et al. used the same idea and gave a reasonable trajectory planning for the dumping process [18]. In the research of fixed point tilting pouring machine, Zhu established the relationship between flow and tilting angle [19]. However, the instantaneous model of pouring flow and angular velocity is studied at the present stage, especially in the relationship between the instantaneous flow state of pouring ladle and the shape of ladle. The influence of melt in pouring mouth on pouring flow is too simplistic and fuzzy, and the model is usually effective only for specially designed ladle; the type of ladle commonly used in casting industry is rarely involved. These problems not only hinder the development and promotion of products, but also increase the difficulty and complexity of the flow control.

Considering the high bearing capacity of parallel mechanism, a mobile casting robot is proposed in our research. It can realize the flexibility of pouring equipment with many varieties and small batches with the ability of continuous pouring. This paper focuses on analyzing of the kinematic characteristics of the proposed 4-UPU parallel mechanism and giving the kinematic closed solution to the mechanism. At the same time, a pouring stage model is built on the basis of a commonly ladle in casting production. The influences of the shape of the ladle and the angle of the pouring nozzle on the dumping model are emphatically investigated. Numerical simulations are given to verify the validity of the proposed model. Moreover, the mapping relation between the kinematic model of parallel manipulator and the pouring model of ladle is given.

\section{Structure of Mobile Heavy Load Pouring Robot}

As shown in Figure 1, a hybrid truss type mobile heavy load pouring robot is proposed [20]. The robot is composed of a $4 \mathrm{WD}$ mobile platform, a slewing device, a hoisting device, a forward device, a backward device, a counterweight device, a parallel manipulator, and a ladle. The $4 \mathrm{WD}$ mobile platform

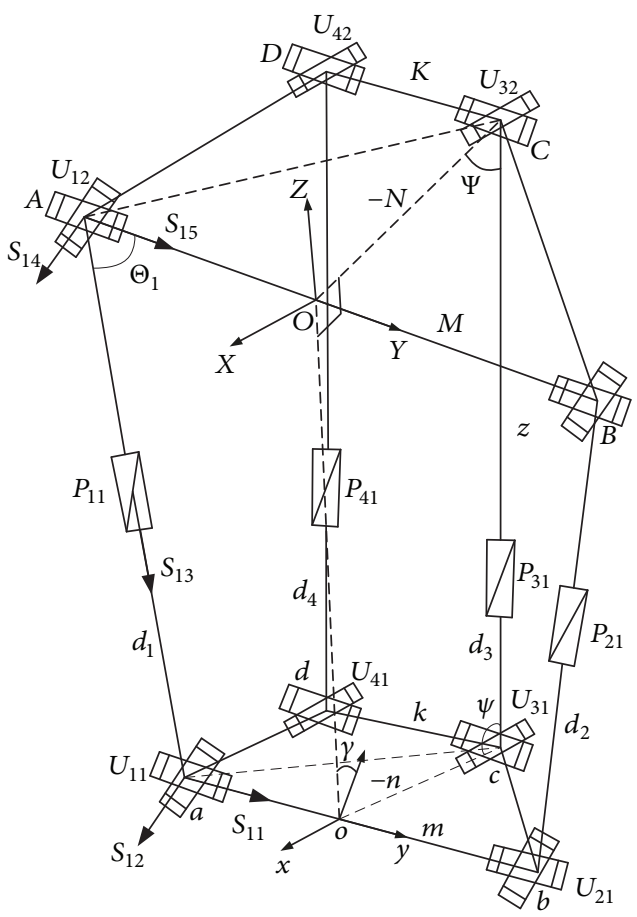

FIGURE 2: Schematic diagram of 4-UPU parallel manipulator for pouring robot.

adopts four-wheel drive plus four-angle stationary support system to realize long distance flexible and stable walking and stationary self-balancing support, so as to improve the stability of operation support. The robot body has $6 \mathrm{DOF}$ in space, three translations, and three rotations. The slewing device and the hoisting device can realize the rotation and the lifting adjustment, respectively. The 4 DOF parallel manipulator can be adjusted to the ladle. The design dimension of the pouring robot is $2150 \mathrm{~mm} \times 1200 \mathrm{~mm} \times 2000 \mathrm{~mm}$. The maximum pouring quantity is $60 \mathrm{~kg}$. Under normal pouring condition, the maximum forward and backward movement range of ladle is about $100 \mathrm{~mm}$, right and left movement is $50 \mathrm{~mm}$, and the vertical elevator height is about $400 \mathrm{~mm}$.

Because the ladle in the process of pouring is around a certain axis rotation, and in order to adapt to the complex pouring environment, we must ensure that the ladle will not slosh in any other direction. Therefore, the parallel unit is designed to be a 4-DOF parallel mechanism with 1-axis rotation and 3-axis translation. Based on the analysis of 3UPU parallel mechanism (3-DOF) [21], a new type of 4-UPU parallel mechanism is proposed to meet the requirements of the pouring operation.

\section{Analysis of Instantaneous Motion Characteristics of 4-UPU Parallel Mechanism}

3.1. Description of Parallel Mechanisms. As shown in Figure 2, the parallel manipulator of the heavy load pouring robot can be simplified as 4-UPU ( $U$ on behalf of a universal joint, $P$ on behalf of a shifting pair) parallel mechanism. The 
upper platform is the fixed platform, installed in the heavy pouring robot beam; the lower platform is a moving platform and is connected with the ladle. The 4 branches are UPU structural kinematic chains. Initial assembly time, the plane $A B a b$ formed by chains 1 and 2, and the upper and lower platforms are vertical to the fixed platform $A B C D$, and the angle between the moving platform $a b c d$ and the plane $A B a b$ is $\gamma$. The plane $C D d c$ formed by chains 3 and 4 and the upper and lower platforms, the angle between the fixed platform $A B C D$ and the plane $C D d c$ is $\Psi$, and the angle between the moving platform $A B C D$ and the plane $C D d c$ is $\psi$. The fixed coordinate system $\boldsymbol{O}-\boldsymbol{X Y Z}$ is located at the midpoint $\mathrm{AB}$ edge, $Z$ axis is perpendicular to the fixed platform, $Y$ axis and $A B$ edge overlap, $C$ points in $\boldsymbol{X}$ axis extension line, the moving coordinate system $\boldsymbol{o}-\boldsymbol{x y} \boldsymbol{z}$ is located at the midpoint $a b$ edge, $\boldsymbol{z}$ axis is perpendicular to the moving platform, $y$ axis and $a b$ edge overlap, $c$ points in $\boldsymbol{x}$ axis extension line, and the $\boldsymbol{y}$ axis is parallel to the $\boldsymbol{Y}$ axis. $o b$ length is $m, o c$ length is $n$, dc length is $k, O B$ length is $M, O C$ length is $N, D C$ length is $K . A a, B b$, $C c$, and $D d$ are $d_{1}, d_{2}, d_{3}$, and $d_{4}$, respectively.

The $U$ pair is composed of $R$ pairs perpendicular to the two axes ( $R$ represents revolute joint), while kinematic chains UPU are equivalent to combinations of RRPRR joints. The axes of each chain kinematic pair are successively represented as $\mathbf{S}_{i 1}, \mathbf{S}_{i 2}, \mathbf{S}_{i 3}, \mathbf{S}_{i 4}, \mathbf{S}_{i 5},(i=1,2,3,4$, for the simple diagram, only the axis of the chain 1 is marked). $\mathbf{S}_{i 1}$ is consistent with the direction of $\mathbf{y}$ axis, $\mathbf{S}_{i 5}$ consistent with the direction of the $\mathbf{Y}$ axis, the angle between $\mathbf{S}_{i 3}$ and $\mathbf{S}_{i 4}$ for $\Theta_{i}, \mathbf{S}_{i 1} \perp \mathbf{S}_{i 2}$, $\mathbf{S}_{i 4} \perp \mathbf{S}_{i 5}, \mathbf{S}_{12} / / \mathbf{S}_{22}, \mathbf{S}_{32} / / \mathbf{S}_{42}$.

3.2. Instantaneous Motion Analysis. The screw theory is used to analyze the motion characteristics. According to the literature [22], the screw is usually expressed as

$$
\mathbf{\$}=\left[\begin{array}{c}
\{\mathbf{S}\}^{T} \\
\{\mathbf{r} \times \mathbf{S}+h \mathbf{S}\}^{T}
\end{array}\right],
$$

where $\mathbf{S}$ represents the $\mathbf{\$}$ axis vector, $\mathbf{r}$ represents the vector of any point on the $\$$ axis to the reference coordinate system, and $h$ is parallel to $\$$, representing the $\$$ axial pitch of $\mathbf{S}$. When $h$ is 0 , the screw is reduced to a line vector that can be used to represent a revolute joint or a binding force. When $h$ is infinite, $\$=(0 ; S)$, the screw degenerates into a couple, which can be used to represent a moving pair or a constraint couple.

When $\$_{1} \circ \$_{2}=\mathbf{S}_{1} \bullet\left(\mathbf{r}_{2} \times \mathbf{S}_{2}+h_{2} \mathbf{S}_{2}\right)+\mathbf{S}_{2} \bullet\left(\mathbf{r}_{1} \times \mathbf{S}_{1}+h_{1} \mathbf{S}_{2}\right)=$ $0, \boldsymbol{\$}_{1}$ and $\boldsymbol{\$}_{2}$ are called reciprocal, and the reciprocal screw is denoted as $\mathbf{\$} \mathbf{r}$. The symbol "o" means reciprocal product. The physical meaning of the reciprocal product reflects the work done by the force screw in the kinematics screw. The reciprocal product is zero, at which point the force screw acts as a constraint on the moving object, whereas the force screw is equivalent to doing work on the moving object.

The kinematic screw of each chain of the parallel mechanism can be obtained from Figure 2:

$$
\begin{aligned}
& \$_{11}=\left(\begin{array}{llllll}
0 & 1 & 0 ; & \sin \Theta_{1} & 0 & 0
\end{array}\right) \\
& \$_{12}=\left(\begin{array}{llllll}
1 & 0 & 0 ; & 0 & -\sin \Theta_{1} & m
\end{array}\right) \\
& \$_{13}=\left(\begin{array}{llllll}
0 & 0 & 0 ; & 0 & \cos \Theta_{1} & -\sin \Theta_{1}
\end{array}\right) \\
& \$_{14}=\left(\begin{array}{llllll}
1 & 0 & 0 ; & 0 & 0 & M
\end{array}\right) \\
& \$_{15}=\left(\begin{array}{llllll}
0 & 1 & 0 ; & 0 & 0 & 0
\end{array}\right) \\
& \$_{21}=\left(\begin{array}{llllll}
0 & 1 & 0 ; & \sin \Theta_{2} & 0 & 0
\end{array}\right) \\
& \$_{22}=\left(\begin{array}{llllll}
1 & 0 & 0 ; & 0 & -\sin \Theta_{2} & -m
\end{array}\right) \\
& \$_{23}=\left(\begin{array}{lllll}
0 & 0 & 0 ; & 0 & -\cos \Theta_{1}-\sin \Theta_{1}
\end{array}\right) \\
& \$_{24}=\left(\begin{array}{llllll}
1 & 0 & 0 ; & 0 & 0 & -M
\end{array}\right) \\
& \$_{25}=\left(\begin{array}{llllll}
0 & 1 & 0 ; & 0 & 0 & 0
\end{array}\right) \\
& \$_{31}=\left(\begin{array}{llllll}
0 & 1 & 0 ; & d_{3} \sin \Psi & 0 & -N+d_{3} \cos \Psi
\end{array}\right)
\end{aligned}
$$

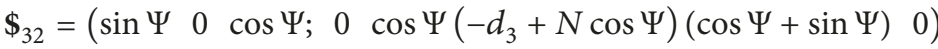

$$
\begin{aligned}
& \$_{33}=\left(\begin{array}{llllll}
0 & 0 & 0 ; & \cos \Psi & 0 & -\sin \Psi
\end{array}\right)
\end{aligned}
$$

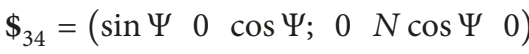

$$
\begin{aligned}
& \$_{35}=\left(\begin{array}{llllll}
0 & 1 & 0 ; & 0 & 0 & -N
\end{array}\right) \\
& \$_{41}=\left(\begin{array}{lllllll}
0 & 1 & 0 ; & d_{3} \sin \Psi & 0 & -N+d_{3} \cos \Psi
\end{array}\right)
\end{aligned}
$$




$$
\begin{aligned}
& \left.\$_{42}=\left(\begin{array}{lllll}
\sin \Psi & 0 & \cos \Psi ; & k \cos \Psi & \cos \Psi\left(-d_{3}+N \cos \Psi\right.
\end{array}\right)(\cos \Psi+\sin \Psi)-k \cos \Psi\right)
\end{aligned}
$$

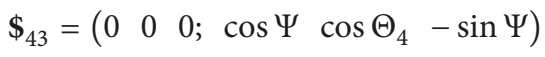

$$
\begin{aligned}
& \$_{44}=\left(\begin{array}{lllll}
\sin \Psi & 0 & \cos \Psi ; K \cos \Psi & N \cos \Psi & -K \sin \Psi
\end{array}\right) \\
& \$_{45}=\left(\begin{array}{llllll}
0 & 1 & 0 ; & 0 & 0 & -N
\end{array}\right)
\end{aligned}
$$

The reciprocal screw of each chain is

$$
\begin{aligned}
& \$_{1}^{r}=\$_{2}^{r}=\left(\begin{array}{llllll}
0 & 0 & 0 ; & 0 & 0 & 1
\end{array}\right) \\
& \$_{3}^{r}=\$_{4}^{r}=\left(\begin{array}{llllll}
0 & 0 & 0 ; & -\cos \Psi & 0 & \sin \Psi
\end{array}\right)
\end{aligned}
$$

From formula (3), it can be seen that the parallel mechanism of the design has 2 over constraints; the kinematic screw system of the 4-UPU parallel mechanism can be obtained by solving the reciprocal screw of (3):

$$
\mathbf{\$}_{f}=\left(\begin{array}{cccccc}
0 & 0 & 0 ; & 1 & 0 & 0 \\
0 & 0 & 0 ; & 0 & 1 & 0 \\
0 & 0 & 0 ; & 0 & 0 & 1 \\
0 & 1 & 0 ; & 0 & 0 & 0
\end{array}\right)
$$

Formula (4) shows that when the initial assembly is performed, the parallel mechanism has three degrees of freedom and a rotational degree of freedom along the $\mathbf{Y}$ direction.

The degree of freedom of the parallel mechanism can be obtained by using the modified G-K formula [23]:

$$
\begin{aligned}
M & =d(n-g-1)+\sum_{i=1}^{g} f_{i}+v \\
& =6(18-20-1)+20+2=4
\end{aligned}
$$

In (5), $d$ is the order of the mechanism, $d=6-\lambda, \lambda$ is the number of common constraint mechanisms, the number of common constraints parallel mechanism in the paper is 0 , $n$ is the number of components, $g$ is the number of kinematic pairs, and $f_{i}$ is the degree of freedom of the $i$ component, $v$ is the total number of over constraints of the mechanism. The over constraints number of the parallel mechanism in this paper is 2 .

It can be seen from formulas (4) and (5) that the analysis results of instantaneous motion characteristics are consistent with the number of degrees of freedom of the mechanism. At the same time, it can be obtained that, under the established coordinate system, the axis relation of each branch chain is invariable, and the number of public constraints and redundant constraints of the mechanism is fixed, so the characteristics of instantaneous motion mechanism are global, and parallel mechanism is designed to meet the needs of pouring operation.

\subsection{Judgment of Drive Joint}

Scenario 1 (4P). Selecting the shifting pair in the 4 chains as the drive joint, according to the literature [24] described in the drive subrule, four mobile pairs are rigidized to get a new 4-UU mechanism, and the kinematic screw of the parallel mechanism is calculated as

$$
\$_{f(4 \mathrm{P})}=\left(\begin{array}{llllll}
0 & 1 & 0 ; & 0 & 0 & 0
\end{array}\right)
$$

According to formula (6), when the 4 shifting pairs are rigid, the new 4-UU mechanism holds a rotational degree of freedom along the $\mathbf{Y}$ axis, and the preselected 4 shifting pairs cannot be the drive pairs of the parallel mechanism at the same time.

Scenario $2(3 \mathrm{P}+1 \mathrm{R})$. The shifting pairs of chains 1,2 , and 3 are selected as drive joints. The $\mathbf{S}_{45}$ axis of the chain 4 is the drive pair, the 4 drive pairs are rigid, and the new parallel mechanism is 3 UU-UPR. The kinematic screw of the parallel mechanism is calculated as follows:

$$
\mathbf{\$}_{f(3 \mathrm{P}+1 \mathrm{R})}=\left(\begin{array}{llllll}
0 & 0 & 0 ; & 0 & 0 & 0
\end{array}\right)
$$

According to formula (7), the new 3UU-UPR mechanism has a degree of freedom of 0 , and the preselected 3 shifting pairs and 1 revolute joint can simultaneously be used as the drive pairs of the parallel mechanism. Therefore, Scenario 2 is chosen as the parallel mechanism drive arrangement mode, which makes the designed 4-UPU parallel mechanism obtain the forward and inverse kinematics, which is beneficial to the mechanism control.

\section{Position Solution of 4-UPU Parallel Mechanism}

First of all, for the further analysis of the chain 4, Section 3.3 of this paper takes the $S_{45}$ axis as the driving pair. Therefore, the $\mathbf{S}_{45}$ axis is rigid and the chain 4 is degenerated into UPR structure. The kinematic screw of the chain is analyzed:

$$
\$_{f(\mathrm{UPR})}=\left(\begin{array}{cccccc}
0 & 0 & 0 ; & 1 & 0 & 0 \\
0 & 0 & 0 ; & 0 & 1 & 0 \\
0 & 0 & 0 ; & 0 & 0 & 1 \\
1 & 0 & 0 ; & 0 & 0 & 0
\end{array}\right)
$$

When the chain $S_{45}$ is rigid, the chain has 4 DOFs, including the freedom of movement of the moving platform, 


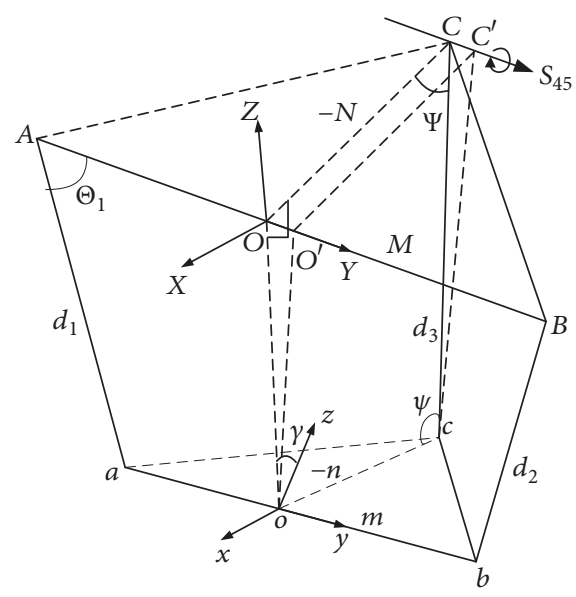

FIgURE 3: 4-UPU equivalent kinematic model.

indicating that the chain 4 does not interfere with the moving motion of the moving platform, but to control the rotation of the moving platform. The $\mathbf{P}_{41}$ of the chain 4 is only forced motion. Therefore, the chain 4 can be regarded as the aided chain. In the process of solving the positive kinematics, the chain 4 is omitted, and a virtual rotation along the $S_{45}$ direction is added at the $C$ point only. As shown in Figure 3. The forward kinematics of the 4-UPU mechanism is finally equivalent to the $o$ coordinates of the midpoint of the AB edge of the three-prism ABCD-abcd.

4.1. Forward Kinematics. When 4-UPU has three translational and one rotational degrees of freedom, the equivalent kinematic model of Figure 3 can be divided into two planes along $\mathrm{Oo}$ and $C c$, respectively, $A B b a$ and $O C c o$ in the $X Z$ plane projection plane $O^{\prime} C^{\prime} c o$, as shown in Figure 4 .

For planar $A B b a$, the $A B$ is always parallel to the $a b$ because the mechanism does not have the rotation along the $\mathbf{X}$ and $\mathbf{Z}$ axes. The relationship can be obtained by trapezoid geometry:

$$
\begin{gathered}
\alpha=\operatorname{acrcos} \frac{d_{2}{ }^{2}-d_{1}{ }^{2}}{2 \sqrt{2} \sqrt{d_{1}^{2}+d_{2}{ }^{2}-2(m-M)^{2}}} \\
\text { Oo }=\frac{\sqrt{d_{1}^{2}+d_{2}^{2}-2(m-M)^{2}}}{\sqrt{2}}
\end{gathered}
$$

$O^{\prime} o$ and $C^{\prime} c$ in the projection plane $O^{\prime} C^{\prime} c o$ of $O C c o$ can be obtained by trigonometric function, respectively:

$$
\begin{aligned}
& O^{\prime} o=\mathrm{Oo} \cdot \sin \alpha \\
& C^{\prime} c=\sqrt{d_{3}^{2}-\left(\mathrm{Oo} \cdot \cos \alpha_{3}\right)^{2}}
\end{aligned}
$$

At this time, $O^{\prime} C^{\prime}, C^{\prime} c, c o, O o$, and $\Psi^{\prime}$ are known in the projection plane $O^{\prime} C^{\prime} \mathrm{co}$; in order to find the moving platform of $o$ position and attitude, $\Gamma^{\prime}$ and $\gamma^{\prime}$ can only demand.
Connect $O^{\prime}$ and $C$, and the can be obtained by the triangle cosine theorem:

$$
\begin{aligned}
O^{\prime} c & =\sqrt{N^{2}+C^{\prime} c^{2}-2 N \cdot C^{\prime} c \cdot \cos \Psi^{\prime}} \\
\gamma^{\prime} & =\operatorname{acrcos}\left(\frac{n^{2}+O^{\prime} o^{2}-O^{\prime} c^{2}}{2 n \bullet O^{\prime} o}\right) \\
\angle o O^{\prime} c & =\operatorname{acrcos}\left(\frac{O^{\prime} o^{2}+O^{\prime} c^{2}-n^{2}}{2 O^{\prime} o \cdot O^{\prime} c}\right) \\
\angle C^{\prime} O^{\prime} c & =\operatorname{acrcos}\left(\frac{N^{2}+O^{\prime} c^{2}-C^{\prime} c^{2}}{2 N \cdot O^{\prime} c}\right)
\end{aligned}
$$

From (13), (14) to angle $\Gamma^{\prime}$,

$$
\Gamma^{\prime}=\angle o O^{\prime} c+\angle C^{\prime} O^{\prime} c
$$

By (9), (12), and (15), we obtain the positive solutions of the 4-UPU parallel mechanism at last:

$$
\begin{aligned}
& x=- \text { Oo } \bullet \sin \alpha \cdot \cos \Gamma^{\prime} \\
& y=\text { Oo } \bullet \cos \alpha \\
& z=-O o \cdot \sin \alpha \cdot \sin \Gamma^{\prime} \\
& \theta=180^{\circ}-\Gamma^{\prime}-\gamma^{\prime}
\end{aligned}
$$

4.2. Inverse Kinematics. The inverse kinematics is given structural parameters and moving platform pose $(x, y, z, \theta)$; then the solution of the 4 inputs $\left(d_{1}, d_{2}, d_{3}, \Psi^{\prime}\right)$ is obtained. According to the coordinate transformation relation, the following equations can be established:

$$
\left(X_{j}, Y_{j}, Z_{j}\right)=\mathbf{T}\left(x_{j}, y_{j}, z_{j}\right)
$$

Among them, $j=a, b, c$. $\mathbf{T}$ is the homogeneous transform matrix:

$$
\mathbf{T}=\left(\begin{array}{cccc}
\cos \theta & 0 & \sin \theta & x \\
0 & 1 & 0 & y \\
-\sin \theta & 0 & \cos \theta & z \\
0 & 0 & 0 & 1
\end{array}\right)
$$

When the structural parameters of the moving platform and the fixed platform are determined, the coordinate of the $j$ point in the fixed coordinate system $\boldsymbol{O}-\boldsymbol{X Y Z}$ can be obtained by formula (17). The input equations of the mechanism are

$$
\begin{aligned}
& d_{1}=\sqrt{\left(X_{A}-X_{a}\right)^{2}+\left(Y_{A}-Y_{a}\right)^{2}+\left(Z_{A}-Z_{a}\right)^{2}} \\
& d_{2}=\sqrt{\left(X_{B}-X_{b}\right)^{2}+\left(Y_{B}-Y_{b}\right)^{2}+\left(Z_{B}-Z_{b}\right)^{2}} \\
& d_{3}=\sqrt{\left(X_{C}-X_{c}\right)^{2}+\left(Y_{C}-Y_{c}\right)^{2}+\left(Z_{C}-Z_{c}\right)^{2}} \\
& \Psi^{\prime}=\arccos \frac{\mathbf{C}^{\prime} \mathbf{O}^{\prime} \cdot \mathbf{C}^{\prime} \mathbf{c}}{\left\|\mathbf{C}^{\prime} \mathbf{O}^{\prime}\right\|\left\|\mathbf{C}^{\prime} \mathbf{c}\right\|}
\end{aligned}
$$




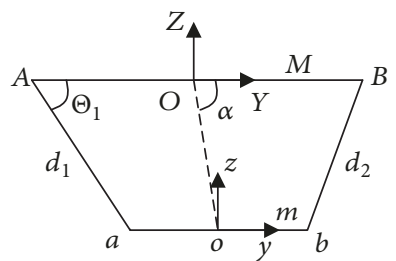

(a) Planar $A B b a$

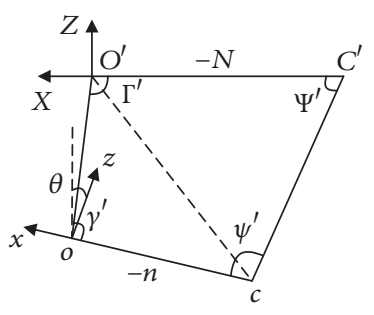

(b) Projective plane $O^{\prime} C^{\prime} c o$

FIGURE 4: The segmentation plane of equivalent kinematic model.

TABLE 1: Inverse kinematics solution of 4-UPU.

\begin{tabular}{lccc}
\hline $\begin{array}{l}X \\
(\mathrm{~mm})\end{array}$ & $\begin{array}{c}y \\
(\mathrm{~mm})\end{array}$ & $\begin{array}{c}z \\
(\mathrm{~mm})\end{array}$ & $\begin{array}{c}\theta \\
\left.{ }^{\circ}\right)\end{array}$ \\
\hline 0 & 0 & -625 & -28 \\
-50 & -50 & -625 & -28 \\
-50 & -50 & -625 & 62 \\
50 & 50 & -625 & 62 \\
\hline$d_{1}$ & $d_{2}$ & $d_{3}$ & $\Psi^{\prime}$ \\
$(\mathrm{mm})$ & $(\mathrm{mm})$ & $(\mathrm{mm})$ & $(\mathrm{mm})$ \\
\hline 626.547 & 626.547 & 872.906 & 1.149 \\
627.026 & 634.004 & 855.101 & 1.202 \\
627.026 & 634.004 & 551.715 & 0.584 \\
634.004 & 627.026 & 637.245 & 0.497 \\
\hline
\end{tabular}

TABLE 2: Kinematics positive solution of 4-UPU.

\begin{tabular}{lccc}
\hline $\begin{array}{l}d_{1} \\
(\mathrm{~mm})\end{array}$ & $\begin{array}{c}d_{2} \\
(\mathrm{~mm})\end{array}$ & $\begin{array}{c}d_{3} \\
(\mathrm{~mm})\end{array}$ & $\begin{array}{c}\Psi^{\prime} \\
(\mathrm{mm})\end{array}$ \\
\hline 626.547 & 626.547 & 872.906 & 1.149 \\
627.026 & 634.004 & 855.101 & 1.202 \\
627.026 & 634.004 & 551.715 & 0.584 \\
634.004 & 627.026 & 637.245 & 0.497 \\
\hline$x$ & $y$ & $z$ & $\theta$ \\
$(\mathrm{mm})$ & $(\mathrm{mm})$ & $(\mathrm{mm})$ & $\left.{ }^{\circ}\right)$ \\
\hline $8.7047 \times 10^{-12}$ & 0 & -625 & -27.983 \\
-50 & -50 & -625 & -27.983 \\
-50 & -50 & -625 & 61.968 \\
50 & 50 & -625 & 61.968 \\
\hline
\end{tabular}

In formula (19), $\mathbf{C}^{\prime} \mathbf{O}^{\prime}$ is the vector representation of the $C^{\prime}$ point in the fixed base coordinate system, and $C^{\prime} \mathbf{c}$ is the vector representation of the length of the projection $\operatorname{rod} C^{\prime} c$ in the fixed coordinate system.

4.3. Numerical Examples of Positive and Inverse Solutions. Set the fixed platform $M=374 \mathrm{~mm}, N=680 \mathrm{~mm}$, the moving platform $m=330 \mathrm{~mm}, n=365 \mathrm{~mm}$; then the corresponding kinematics inverse kinematics solution is shown in Table 1, and the corresponding forward kinematic solutions are shown in Table 2.

As can be seen from Tables 1 and 2, the kinematics forward and inverse solution equations are closed, which shows

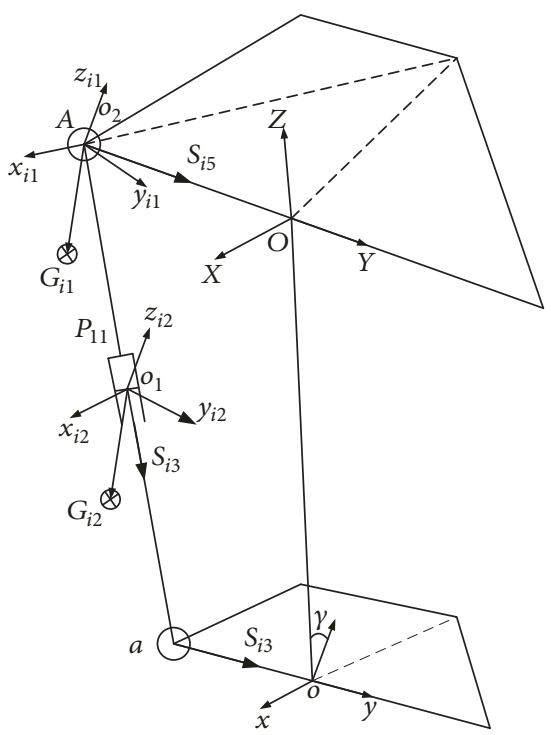

FIGURE 5: Coordinate systems for a leg and moving platform.

that the algorithm in this paper is correct and feasible. Compared with algebraic method, the unique solution in a given workspace can be obtained directly.

4.4. Inverse Dynamics. The dynamics of the 4-UPU parallel mechanism of the pouring robot is shown in Figure 5 Since each leg, only a cylinder, can be assumed to be composed of two segments, two parallel local systems, $i_{1}$-frame and $i_{2}$ frame attached to the upper segment 1 and lower segment 2 , respectively, of the leg $i$, are defined with their corresponding origins $o_{i 1}$ and $o_{i 2} . G_{i 1}$ and $G_{i 2}$ are the center of gravity of the upper and lower segments. The position vector of the center of mass of each part is written as

$$
\begin{aligned}
& \mathbf{r}_{o}=\text { Oo }+{ }_{o}^{O} \mathbf{R} \boldsymbol{\rho}_{o} \\
& \mathbf{r}_{i 1}=\mathbf{S}_{i 1}+{ }_{o}^{O} \mathbf{R} \boldsymbol{\rho}_{i 1} \\
& \mathbf{r}_{i 2}=\mathbf{S}_{i 1}+{ }_{i}^{O} \mathbf{R S} \mathbf{S}_{i 3}+{ }_{i}^{O} \mathbf{R} \boldsymbol{\rho}_{i 2}
\end{aligned}
$$

$\boldsymbol{\rho}_{i 1}, \boldsymbol{\rho}_{i 2}$ are the position vectors of $G_{i 1}$ and $G_{i 2}$ in the local coordinate system, respectively, $\boldsymbol{\rho}_{o}$ is the position vector of the $a$ point and the moving platform centroid, $\boldsymbol{\rho}_{i 1}, \boldsymbol{\rho}_{i 2}, \boldsymbol{\rho}_{o}$ are all constant vectors, $\mathbf{S}_{i 3}=S_{i 3} \mathbf{s}_{i 3}, \mathbf{s}_{i 3}$ is the direction vector of the shifting axis, and $S_{i 3}$ is the length of the shifting axis. The 
absolute velocities of the masses on each body are given by differentiating (20) through (22) as

$$
\begin{aligned}
\dot{\mathbf{r}}_{o} & =\dot{\mathbf{O} o}+{ }_{o}^{O} \mathbf{R} \widetilde{\boldsymbol{\omega}}_{o} \boldsymbol{\rho}_{o} \\
\dot{\mathbf{r}}_{i 1} & ={ }_{i}^{O} \mathbf{R} \widetilde{\boldsymbol{\omega}}_{i} \boldsymbol{\rho}_{i 1} \\
\dot{\mathbf{r}}_{i 2} & ={ }_{o}^{O} \mathbf{R} \widetilde{\boldsymbol{\omega}}_{i} \mathbf{S}_{i 3}+{ }_{i}^{O} \mathbf{R} \dot{\boldsymbol{S}}_{i 3}+{ }_{i}^{O} \mathbf{R} \widetilde{\boldsymbol{\omega}}_{i} \boldsymbol{\rho}_{i 2}
\end{aligned}
$$

$\dot{\mathbf{S}}_{i 3}, \boldsymbol{\omega}_{i}, \ddot{\mathbf{S}}_{i 3}, \dot{\boldsymbol{\omega}}_{i}$ can be obtained by solving two differentials of the "Oo $+{ }_{o}^{O} \mathbf{R S} \mathbf{S}_{i 5}=\mathbf{S}_{i 1}+{ }_{i}^{O} \mathbf{R S}_{i 3}$ " of the vector-loop equation of the parallel mechanism.

The total kinetic energy and potential energy of the new 4-UPU parallel mechanism can be written as

$$
\begin{aligned}
T= & \frac{1}{2} \int \dot{\mathbf{r}}_{o}^{T} \dot{\mathbf{r}}_{0} d m_{o} \\
& +\sum_{i=1}^{4} \frac{1}{2}\left(\int \dot{\mathbf{r}}_{i 1}^{T} \dot{\mathbf{r}}_{i 1} d m_{i 1}+\int \dot{\mathbf{r}}_{i 2}^{T} \dot{\mathbf{r}}_{i 2} d m_{i 1}\right) \\
U= & \frac{1}{2} \int-\mathbf{g}^{T} \mathbf{r}_{0} d m_{o} \\
& +\sum_{i=1}^{4} \frac{1}{2}\left(\int\left(-\mathbf{g}^{T}\right) \mathbf{r}_{i 1} d m_{i 1}+\int\left(-\mathbf{g}^{T}\right) \mathbf{r}_{i 2} d m_{i 2}\right)
\end{aligned}
$$

In (24) and (25), $\mathbf{g}$ is the gravitational acceleration vector, and $m_{o}, m_{i 1}$, and $m_{i 2}$ are the mass of the moving platform, the upper segment, and the lower segment, respectively.

Using the Lagrange equation, the dynamic equation of the new 4-UPU parallel mechanism can be written as

$$
\tau=\frac{d}{d t}\left(\frac{\partial L}{\partial \dot{q}}\right)-\frac{\partial L}{\partial q},
$$

where $L=T-U$, generalized coordinates $q=$ $\left[\begin{array}{llll}S_{13} & S_{23} & S_{33} & \theta_{4}\end{array}\right]^{T}$, and the driving force and driving torque $\tau=\left[\begin{array}{llll}f_{1} & f_{2} & f_{3} & T_{4}\end{array}\right]^{T}$.

\section{Whole Cycle Tilting Prediction Model for Ladle}

The goal of the mobile heavy load pouring robot is to complete the continuous casting of many varieties and small batches. The pouring flow rate is one of the key factors affecting the quality of the castings and also the basis for the movement of the new 4-UPU parallel mechanism. Therefore, it is necessary to establish the pouring model of the ladle to realize the dumping movement of the parallel mechanism. Noda and Terashima established the flow rate model and feedback control in the study of the flow control of the pouring robot [25]. This paper focuses on the volume change of the ladle in the pouring process and then establishes the model of pouring angular velocity and pouring flow.

5.1. Ladle Model. In order to control the moving platform movement of the parallel pouring robot and realize constant current control of pouring flow, it is necessary to establish

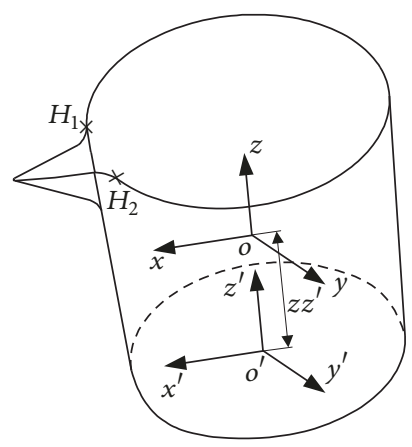

FIgURE 6: Inner cavity model of ladle.

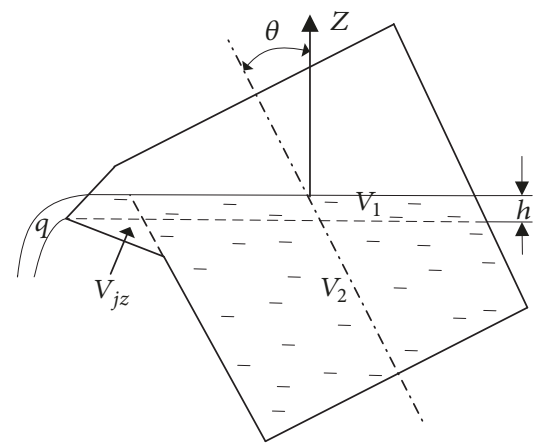

Figure 7: Sectional view of $x^{\prime} z^{\prime}$ surface in ladle inner cavity.

the relationship between the pouring angle of the ladle and the instantaneous change of the pouring flow rate, which can maintain basic constant outflow of ladle pouring liquid flow.

In this paper, pouring ladle selects the steel ladle which is common in the casting foundry [26]. The shape of the inner cavity of the ladle is shown in Figure 6, similar to frustum of a cone. The $\mathbf{o - x y z}$ is the moving platform coordinate system of the parallel mechanism, located in the middle of the inner cavity, $\mathbf{o}^{\prime}-\mathbf{x}^{\prime} \mathbf{y}^{\prime} \mathbf{z}^{\prime}$ is the ladle coordinate system, located at the center of the ladle bottom, $\mathbf{o}^{\prime}-\mathbf{x}^{\prime} \mathbf{y}^{\prime} \mathbf{z}^{\prime}$ can be obtained by translating $\mathbf{0}-\mathbf{x y z}$ along $\mathbf{z}$ axis- $z z^{\prime}$ lengths, and here the pouring mouth and the cone surface two intersection points are marked as $H_{1}, H_{2}$. In order to build the relationship between ladle volume and tilting angle, the section of the ladle is made along the $x^{\prime} z^{\prime}$ plane as shown in Figure 7.

Figure 7 is the flowing state of the pouring liquid in the ladle at the instant when the ladle is poured. $V_{2}$ is the volume of the pouring liquid in the frustum of the cone below the pouring mouth. $V_{j z}$ is the volume of the pouring liquid in the pouring mouth below the pouring mouth. $V_{1}$ is the volume of the pouring liquid above the pouring mouth, $h$ is the height of the pouring liquid above the pouring mouth, $q$ is the pouring flow, and the $\theta$ is the tilting angle.

5.2. Tilting Model. In order to simplify the model, it is assumed that the pouring fluid is an incompressible ideal fluid. Since the shape of the ladle is different in different angle intervals when the pouring angle changes, the segmentation model is established according to the divided angle interval. 


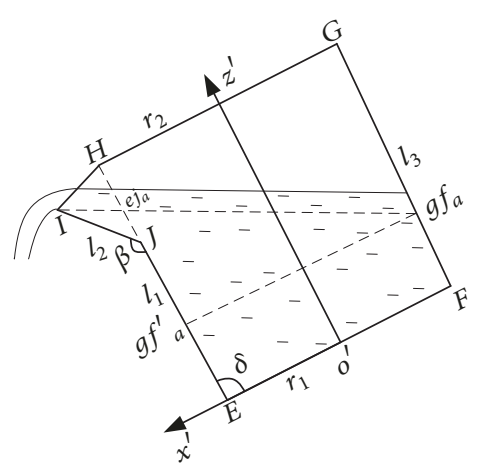

Figure 8: Position of liquid level at Case 1.

Case 1. In Figure 8, the EFGHIJ represents the section of the ladle along the $x^{\prime} z^{\prime}$ plane, $\beta$ indicates the angle between $I J$ and $J E, \delta$ indicates the angle between $E G$ and $J E$, and $e j_{a}$ indicates the intersection point between the level of the pouring mouth $I$ and the $E J$ extension line. $g f_{a}$ indicates the intersection point between the level of the pouring mouth $I$ and the GF, $r_{1}$ represents the bottom radius of the ladle, and $r_{2}$ represents the top radius of the ladle, and the lengths of $J E$, $I J$, and $G F$ are $l_{1}, l_{2}$, and $l 3$, respectively. Figure 8 is the flow state of the casting liquid at the beginning of casting, $V_{1}, V_{2}$, and $V_{j z}$ all exist, and the ladle does not show the bottom. The solution of the volume of each part is as follows.

In order to show the difference, $V_{1}, V_{2}, V_{j z}, h$, and $q$ are labeled as $V_{1 a}, V_{2 a}, V_{j z a}, h_{a}$, and $q_{a}$, respectively, at the time of Case 1.

$V_{2 a}$. Divide $V_{2 a}$ into two parts for the upper and lower through point $g_{f a}, V_{2 a \text { (up) }}$ is wedge cone, $V_{2 a \text { (down) }}$ is a frustum of a cone body, and then the liquid level equation of the pouring mouth $I$ is

$$
z^{\prime}=\tan \theta\left(x^{\prime}-I_{x}\right)-I_{z}
$$

$$
\text { In (27), } I_{x}=r_{1}-l_{1} \cos \delta+l_{2} \cos (\beta-\delta) \text {, }
$$

$$
I_{z}=l_{1} \sin \delta+l_{2} \sin (\beta-\delta) \text {. }
$$

EJ equation:

$$
z^{\prime}=-\tan \delta x^{\prime}+r_{1} \tan \delta
$$

GF equation:

$$
z^{\prime}=\tan \delta x^{\prime}+r_{1} \tan \delta
$$

By the simultaneous equations (27) and (29) and (27) and (30), the intersection points $e j_{a}$ and $g f_{a}$ coordinates can be obtained:

$$
\begin{gathered}
e j_{a}:\left(\begin{array}{c}
\frac{-I_{x}+r_{1} \tan \delta+I_{z} \tan \theta}{\tan \delta+\tan \theta}, \\
\csc (\delta+\theta) \sin \delta\left(I_{z} \cos \theta+\left(r_{1}-I_{x}\right) \sin \theta\right)
\end{array}\right) \\
g f_{a}:\left(\begin{array}{c}
\frac{I_{x}-r_{1} \tan \delta-I_{z} \tan \theta}{\tan \delta-\tan \theta}, \\
\csc (\delta-\theta) \sin \delta\left(I_{z} \cos \theta-\left(r_{1}-I_{x}\right) \sin \theta\right)
\end{array}\right)
\end{gathered}
$$

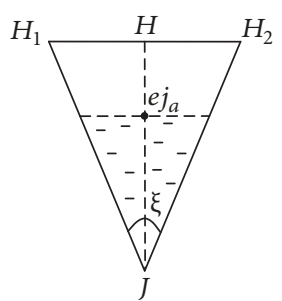

FIGURE 9: The $H_{1} H_{2} J$ face of the pouring mouth triangle.

Surface equation of frustum cone is

$$
x^{\prime 2}+y^{\prime 2}=\left(r_{1}+\frac{r_{2}-r_{1}}{l_{3} \sin \delta} z^{\prime}\right)^{2}
$$

By formulas (31) and (32) and volume integral methods, $V_{2 a(\text { up })}$ can be obtained:

$$
V_{2 a(\text { up })}=\int_{z^{\prime}{ }_{1 a}}^{z^{\prime}{ }_{2 a}} \int_{x_{1 a}^{\prime}}^{x^{\prime}{ }_{2 a}} \int_{y^{\prime}{ }_{1 a}}^{y^{\prime}{ }_{2 a}} d y^{\prime} d x^{\prime} d z^{\prime}
$$

In (33),

$$
\begin{aligned}
& y_{2 a}^{\prime}=-y_{1 a}^{\prime}=\sqrt{\left(r_{1}+\frac{r_{2}-r_{1}}{l_{3} \sin \delta} z^{\prime}\right)^{2}-x^{\prime 2}} \\
& x_{2 a}^{\prime}=\frac{z^{\prime}-r_{1} \tan \delta}{-\tan \delta} \\
& x_{1 a}^{\prime}=\frac{z^{\prime}-I_{z}}{\tan \theta}+I_{x} \\
& z_{2 a}^{\prime}=e j_{a}\left(z^{\prime}\right) \\
& z_{1 a}^{\prime}=g f_{a}\left(z^{\prime}\right)
\end{aligned}
$$

The $e j_{a}\left(z^{\prime}\right)$ and $g f_{a}\left(z^{\prime}\right)$ represent the $z^{\prime}$ coordinates of the intersection points $e j_{a}$ and $g f_{a}$.

$V_{2 a \text { (down) }}$ can be obtained by the volume formula of truncated cone:

$$
V_{2 a(\text { down })}=\frac{1}{3} \pi g f_{a}\left(z^{\prime}\right)\left(R_{2 a}^{2}+r_{1}^{2}+R_{2 a} \cdot r_{1}\right)
$$

In (35),

$$
R_{2 a}=r_{1}+\frac{r_{2}-r_{1}}{l_{3} \sin \delta} g f_{a}\left(z^{\prime}\right)
$$

Add formulas (33) and (35) to get the expression of $V_{2 a}$.

$V_{j z a}$. Because the ladle body taper is generally $1: 10 \sim 1: 15$, pouring mouth angle within $30^{\circ} \sim 40^{\circ}$, and after applying the lining, the size of the nozzle is smaller, so the pouring mouth can be regarded as a triangular body. The $H_{1} H_{2} J$ face of the triangular body is shown in Figure 9. $\xi$ is the angle of $\angle H_{1} J H_{2}$.

According to the principle of similar triangle, the wetted area of the liquid in $H_{1} H_{2} J$ in the triangular can be obtained:

$$
S_{e j_{a}}=\left(l_{3}-l_{1}\right) \tan \frac{\xi}{2} \frac{e j_{a}\left(z^{\prime}\right)-l_{1} \sin \delta}{\sin \delta}
$$


It can be obtained by formula (37) and volume formula of triangular:

$$
V_{j z a}=\frac{1}{3} S_{e j_{a}} h_{I J}
$$

where $h_{I J}$ is $I J$ in the perpendicular line of $H_{1}, h_{I J}=l_{2} \sin \beta$. $V_{1 a}$

$$
V_{1 a} \approx h_{a} \cdot S_{1 a}
$$

$V_{1 a}$ can be approximately written in the form of (39). $S_{1 a}$ is the area of the pouring surface of the over pouring mouth $I$, composed of pouring liquid surface area $S_{2 a}$ of $V_{2 a}$ and $S_{j z a}$ of $V_{j z a} . S_{j z a}=3 V_{j z a} /\left(l_{2} \sin (\beta-\delta-\theta)\right) . S_{2 a}$ is obtained by (31) and (32) and the area projection methods:

$$
S_{2 a}=\frac{\int_{g f_{a}\left(x^{\prime}\right)}^{e j_{a}\left(x^{\prime}\right)} 2 y_{a(\text { tou })}^{\prime} d x^{\prime}}{\cos \theta}
$$

In (40), $e j_{a}\left(x^{\prime}\right)$ and $g f_{a}\left(x^{\prime}\right)$ represent the $x^{\prime}$ coordinates of intersection points $e j_{a}$ and $g f_{a}$,

$$
\begin{aligned}
& y_{a(\mathrm{tou})}^{\prime} \\
& =\sqrt{\left(r_{1}+\frac{\left(r_{2}-r_{1}\right) \csc \delta\left(I_{z}+\left(x^{\prime}-I_{x}\right) \tan \theta\right)}{l_{3}}\right)^{2}-x^{\prime 2}}
\end{aligned}
$$

Because the pouring fluid is incompressible ideal fluid, the flow rate of $q_{a}$ can be obtained by Bernoulli equation

$$
q_{a}=\int_{0}^{h_{a}} \sqrt{2 g h_{a}} d A_{h_{a}}
$$

In (42), $g$ is the gravitational acceleration, and $A_{h_{a}}$ is ladle outflow of the cross section area, which is equal to the projection of the flow velocity direction of the pouring liquid at the exit of the $H$. Its solution is similar to the wetted area of the solution at $\mathrm{H}_{1} \mathrm{H}_{2} J$ with no more detailed description.

$$
A_{h_{a}}=\frac{H_{1} H_{2} \cdot h_{a}^{2}}{2 H I}
$$

In (43), $H_{1} H_{2}$ stands for side length and $H I$ stands for $\triangle H_{1} I H_{2}$ over $H$ point midline length. Simultaneous equations (42), (43) can be obtained:

$$
h_{a}=\left(\frac{H I \cdot q_{a}}{H_{1} H_{2} \sqrt{g}}\right)^{2 / 5}
$$

The equations (40), (44) and $S_{j z a}$ are brought in (39) to obtain $V_{1 a}$.

Case 2. Figure 10 shows that the pouring water in the ladle continues to decrease as the pouring occurs, and the bottom of the ladle has been exposed. At this point, $V_{1}, V_{2}$, and $V_{j z}$ all exist, which is similar to the solution of Case 1. Therefore, Case 2 is solved and only a brief introduction is given.

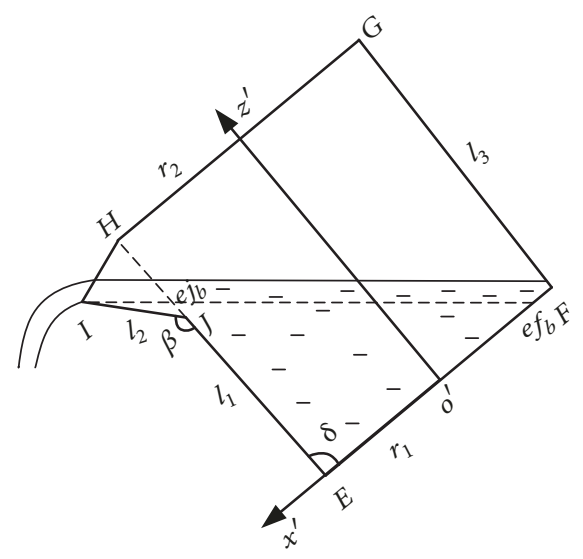

Figure 10: Position of liquid level at Case 2.

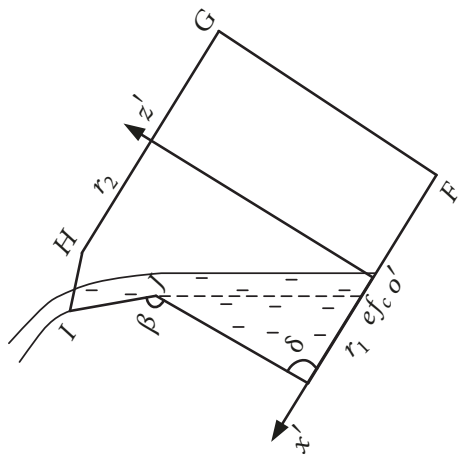

Figure 11: Position of liquid level at Case 3.

In order to show the difference, $V_{1}, V_{2}, V_{j z}, h$, and $q$ are labeled as $V_{1 b}, V_{2 b}, V_{j z b}, h_{b}$, and $q_{b}$ respectively, at the time of Case 2.

$V_{2 b}$. In Case 2, at this point $V_{2 b \text { (down) }}=0$, the intersection point of the pouring mouth $I$ and the $E F$ is

$$
e f_{b}:\left(I_{z} \csc \theta+I_{x}, 0\right)
$$

Then $V_{2 b}$ is

$$
V_{2 b}=V_{2 b \text { (up) }}=\int_{z^{\prime}{ }_{1 b}}^{z^{\prime}{ }_{2 b}} \int_{x^{\prime}{ }_{1 b}}^{x^{\prime}{ }_{2 b}} \int_{y^{\prime}{ }_{1 b}}^{y^{\prime}{ }_{2 b}} d y^{\prime} d x^{\prime} d z^{\prime}
$$

In (46), $z_{1 b}^{\prime}=0$, other upper and lower solutions and forms are the same as Case 1 .

$V_{j z b}$. The solution is exactly the same as Case 1 .

$V_{1 b}$. The solution is exactly the same as Case 1.

Case 3. Figure 11 is the end of the tilting phase, $V_{j z}$ disappears, and $V_{1}$ and $V_{2}$ remain. After pouring the liquid through the point of $J$, the $I J$ falls off a certain distance and then falls. Compared with Cases 1 and 2, this stage is slightly different.

In order to show the difference, $V_{1}, V_{2}, V_{j z}, h$, and $q$ are labeled as $V_{1 b}, V_{2 b}, V_{j z b}, h_{b}$, and $q_{b}$ respectively, at the time of Case 2. 
In order to show the difference, $V_{1}, V_{2}, V_{j z}, h$, and $q$ are labeled as $V_{1 c}, V_{2 c}, V_{j z c}, h_{c}$, and $q_{c}$ respectively, at the time of Case 2.

$V_{2 c}$. The liquid level equation of the overflow pouring mouth $J$ is

$$
z^{\prime}=\tan \theta\left(x^{\prime}-J_{x}\right)-J_{z}
$$

In (47), $J_{x}=r_{1}-l_{1} \cos \delta, J_{z}=l_{1} \sin \delta$.

Then

$$
V_{2 c}=V_{2 c(\text { up })}=\int_{z^{\prime}{ }_{1 c}}^{z_{2 c}^{\prime}} \int_{x_{1 c}^{\prime}}^{x_{2 c}^{\prime}} \int_{y_{1 c}^{\prime}}^{y_{2 c}^{\prime}} d y^{\prime} d x^{\prime} d z^{\prime}
$$

In (48),

$$
\begin{aligned}
& x_{1 c}^{\prime}=\frac{z^{\prime}-J_{z}}{\tan \theta}+J_{x} \\
& z_{1 c}^{\prime}=0 \\
& z_{2 c}^{\prime}=J_{z}
\end{aligned}
$$

Other upper and lower solutions and forms are the same as Case 1.

$V_{1 c}$. Because of the $V_{j z c}=0$ in Case $3, S_{j z a}=0$.

$$
S_{1 c}=S_{2 c}=\frac{\int_{J_{z} \csc \theta+J_{x}}^{J_{x}} 2 y_{c(\text { tou })}^{\prime} d x^{\prime}}{\cos \theta}
$$

In Case 3, the outlet of the pouring is $\triangle H_{1} J H_{2}$, then

$$
A_{h_{c}}=\frac{H_{1} H_{2} \cdot h_{a}^{2}}{2 H J}
$$

1.

$V_{1 c}$ other equations solving methods are the same as Case

At this point, the sectional model of the water flow in the whole cycle is established. As can be seen from the model, each stage is divided according to the angle of the pouring mouth. When the angle of the pouring mouth is too small, Case 2 may disappear, the direct transition to Case 3, and the volume of $V_{2 \text { (down) }}$ will be increased, but the solution is the same. It is also possible that the original pouring volume of the ladle is relatively small, and the pouring process starts directly from Case 2 or Case 3 , but the tilting model established in this paper is still valid, except that the initial flow angle of the ladle is different.

Here are some explanations for the tilting model:

(a) The actual pouring should control the pouring liquid height, avoiding too high pouring liquid to cause safety accidents. The maximum pouring height in the model is related to the length of center lines $H J$ and HI.

(b) The model established in this paper is an ideal fluid model, without considering the influence of viscosity on the flow velocity of the pouring fluid. The viscosity should be considered in the actual pouring and formula (42) should be modified.

(c) The model described in this paper is only applicable to a kind of common pouring ladle, and other kinds of ladle should be analyzed separately.

5.3. Angular Velocity Model. The relationship between volume and flow can be obtained:

$$
q(t)=-\frac{d\left(V_{1}(\theta(t))+V_{2}(\theta(t))+V_{j z}(\theta(t))\right)}{d t}
$$

When (38) is brought into the upper expansion, the tilting velocity $w(t)$ can be obtained:

$$
w(t)=-\frac{q(t)+(d h(t) / d t) S_{2}(\theta(t))}{\left(\partial S_{2}(\theta(t)) / \partial \theta(t)\right) h(t)+\partial V_{1}(\theta(t)) / \partial \theta(t)+\partial V_{2}(\theta(t)) / \partial \theta(t)}
$$
tilting:

Also by $w=d \theta / d t$, we can get the total time needed for

$$
t=\int_{\theta_{1}}^{\theta_{2}} \frac{1}{w} d \theta
$$

In (54), $\theta_{1}$ is the beginning of the tilting angle. $\theta_{2}$ is the end of tilting angle.

\section{Numerical Verification}

6.1. Validation of the Tilting Prediction Model. To verify the correctness of the tilting model, the model is compiled into MATLAB. And the $r_{1}=195 \mathrm{~mm}, r_{2}=212 \mathrm{~mm}, l_{1}=340 \mathrm{~mm}$, $l_{2}=170 \mathrm{~mm}, l_{3}=490 \mathrm{~mm}, \beta=135^{\circ}, \delta=92^{\circ}, \zeta=35^{\circ}$. When pouring, the total volume of pouring liquid in pouring ladle is $V_{\text {all }}=5.8 \times 10^{7} \mathrm{~mm}^{3}$. In order to facilitate verification, the flow rate of the whole pouring process is constant, $q_{1}=$ $2 \times 10^{5} \mathrm{~mm}^{3} / \mathrm{s}, q_{2}=4 \times 10^{5} \mathrm{~mm}^{3} / \mathrm{s}, q_{3}=6 \times 10^{5} \mathrm{~mm}^{3} / \mathrm{s}$, and $h$ is also the fixed value. Before computing, we start with some initialization settings for the model.

(1) Critical Point Judgment. According to the geometric relation, the three phases in Section 5 are easy to obtain. The critical angles are $40.930^{\circ}$ and $42.994^{\circ}$, respectively. The initial flow angle of the pouring liquid is $\theta_{\text {initial }}$, the shed end angle is $\theta_{\text {end }}$, and they can be obtained according to the following formula:

$$
\begin{gathered}
V_{1}+V_{2}+V_{j z}=V_{\text {all }} \\
V_{1 c}+V_{2 c}=q
\end{gathered}
$$


The search method is used to get $\theta_{\text {initial }}=7.222^{\circ}, \theta_{\text {end }}=$ $87.994^{\circ}$. Assuming that the dip angle of the ladle at the beginning of the tilting is $\theta_{1}=0$, the whole tilting stage can be divided into

Initial stage: $0^{\circ} \sim 7.222^{\circ}$

Case 1 stage: $7.222^{\circ} \sim 40.930^{\circ}$

Case 2 stage: $40.930^{\circ} \sim 42.994^{\circ}$

Case 3 stage: $42.994^{\circ} \sim 87.994^{\circ}$

(2) Angular Velocity at Initial Stage. $\theta_{1} \sim \theta_{\text {initial }}$ is the initial stage, this stage without pouring liquid outflow, and no angular velocity model. In order to make the pouring out of the initial angular velocity $w_{\text {initial }}$ change smoothly when the angle reaches $\theta_{\text {initial }}$, the initial stage angular velocity is set to the value, and its value is $w_{\text {initial }}$.

(3) Treatment of Differential Terms. In solving the angular velocity, it is necessary to derivate the volume term, but for the volume $V_{2(\text { up })}$ without analytic solution, only the analytic formula of the second integral in the integral can be solved:

$$
V_{2 \text { (up) }}=\int_{z_{1}^{\prime}{ }_{1}}^{z_{2}^{\prime}} V_{2(\text { up })}\left(z^{\prime}, \theta(t)\right) d z^{\prime},
$$

where $z^{\prime}{ }_{1}$ and $z^{\prime}{ }_{2}$ contain differential terms $\theta(t)$. The derivative of formula (56) about $\theta(t)$ is derived by using parametric integral derivative formula:

$$
\begin{aligned}
\frac{d V_{2 \text { (up) }}}{d \theta(t)}= & \int_{z^{\prime}{ }_{1}}^{z_{2}{ }_{2}} \frac{d V_{2(\text { up })}\left(z^{\prime}, \theta(t)\right)}{d \theta(t)} d z^{\prime} \\
& +V_{2 \text { (up) }}\left(z^{\prime}{ }_{2}, \theta(t)\right) \frac{d z^{\prime}{ }_{2}}{d \theta(t)} \\
& -V_{2 \text { (up) }}\left(z^{\prime}{ }_{1}, \theta(t)\right) \frac{d z^{\prime}}{d \theta(t)}
\end{aligned}
$$

The integral term in (57) is solved by MATLAB numerical integration. $S_{2 c}$ cannot find the analytic solution, and its calculation process is similar to that of $V_{2 \text { (up) }}$.

With the method of (54), the angle and angular velocity change with time, as shown in Figure 12.

In Figures 12(a) and 12(b), respectively, for the instantaneous relationship of tilting angle and angular velocity of the whole period, (a) shows that the changes of tilting cycle angle are relatively smooth, no severe fluctuations; (b) shows that the angular velocity changes are nonlinear during the full tilting period, and the angular velocity changes greatly at the transition of $\mathrm{B}$ and $\mathrm{C}$, which is caused by the disappearance of the $V_{j z}$ volume. As can be seen from Table 3, the error of the theory time $\left(V_{\text {all }} / q_{i}, V_{\text {all }}\right.$ is the total volume of pouring liquid in the ladle. $q_{i}$ is pouring liquid flow) and simulation time of different pouring flow are only $0.8 \sim 2.4 \%$; the error of time was less than $3 \mathrm{~S}$, showing that the model is correct and can be used for real-time control of ladle pouring speed. It is especially suitable for developing tilting control of low cost automatic pouring robot and also can be used as the basic model of tilting closed loop control for high precision automatic pouring robot.
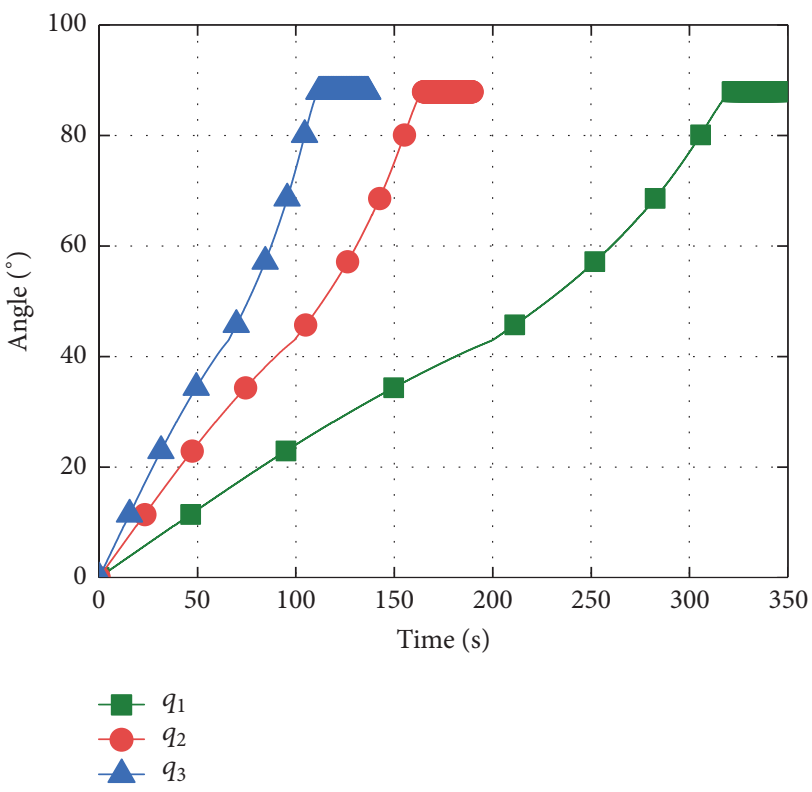

(a)

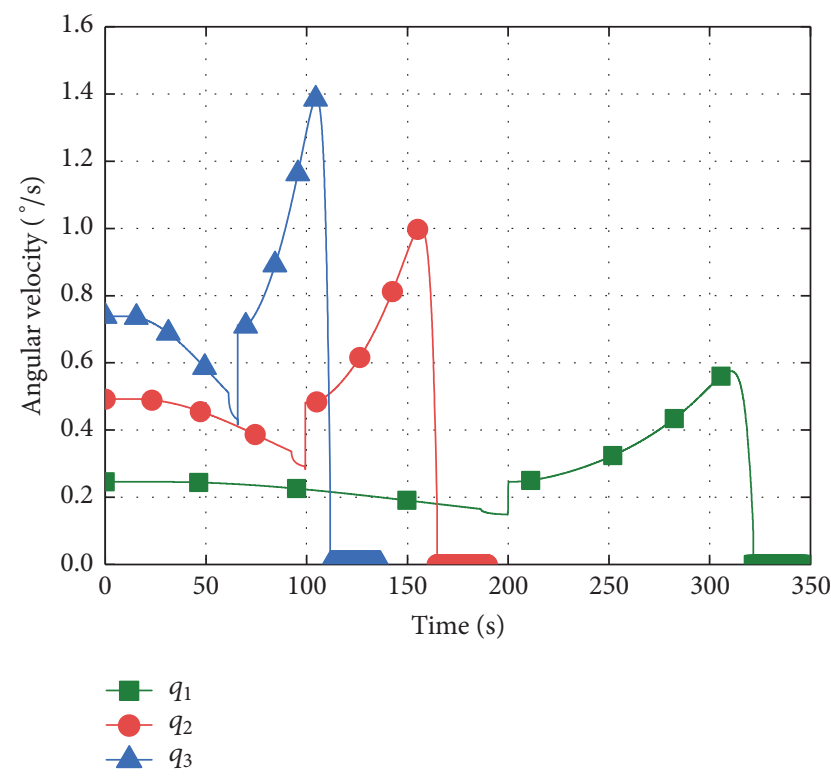

(b)

FIGURE 12: Numerical simulation results of tilting prediction.

TABLE 3: Comparison between dumping theoretical time and simulation time.

\begin{tabular}{lccc}
\hline No. & Theoretical time (s) & Simulation time (s) & Error (\%) \\
\hline$q_{1}$ & 290 & 292.345 & 0.8 \\
$q_{2}$ & 145 & 148.165 & 2.1 \\
$q_{3}$ & 96.667 & 99.020 & 2.4 \\
\hline
\end{tabular}

6.2. Kinematic Simulation of Parallel Mechanism. The pouring process of ladle eventually depends on the designed parallel mechanism, so it is necessary to obtain the change of the driving pair of the parallel mechanism in the whole cycle of the tilting, so as to realize the tilting control of the ladle. In 


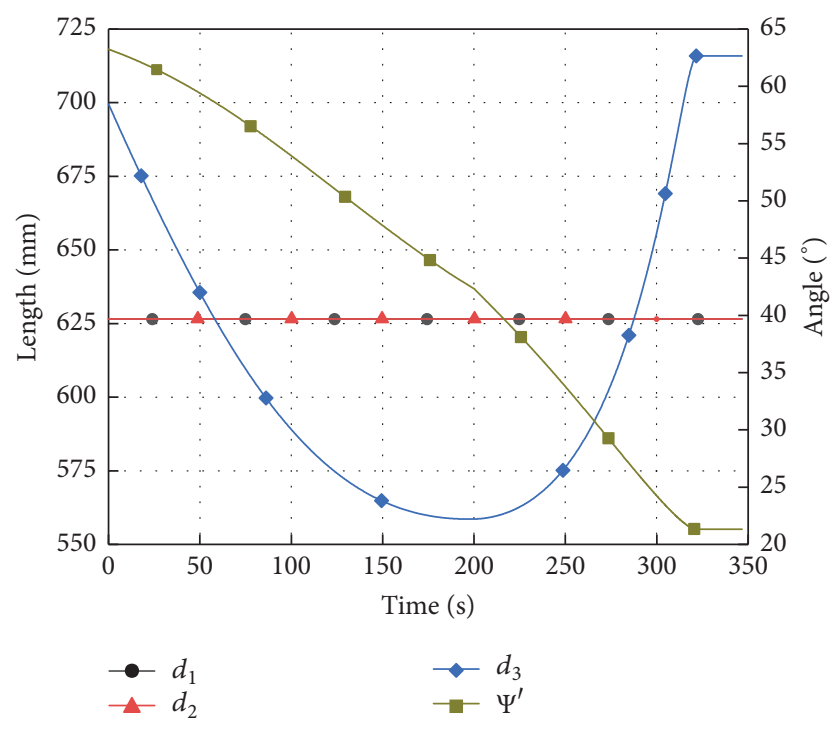

FIGURE 13: Drive pair changed during all the pouring cycle.

this example, the pouring data of the ladle in Section 6.1 at the flow rate of $q_{1}$ are brought into the inverse position model of Section 4.2 parallel mechanism, and the structure parameters are the same as in Section 4.3. For calculation and reflecting the driving pair of ladle pouring control will be simplified, a parallel platform to rotate around the fixed position. The coordinates of $X, y$, and $Z$ are $0 \mathrm{~mm}, 0 \mathrm{~mm}$, and $-625 \mathrm{~mm}$, respectively. The numerical results are shown in Figure 13. In order to simplify the calculation and reflect the tilting control of the driving pair, the parallel platform is set to rotate around the fixed position.

As can be seen from Figure 13, the length of $d_{1}$ and $d_{2}$ rods is fixed throughout the full cycle of tilting, and $d_{3}$ rod and the $\Psi^{\prime}$ axis vary with time. This shows that when pouring in the fixed position, the ladle rotation is realized by the coupling of the $d_{3}$ rod and the $\Psi^{\prime}$ axis, while $d_{1}$ and $d_{2}$ are only used to restrict the space position of the moving platform. The variation of $d_{3}$ rod length is symmetrical in the whole tilting cycle; that is, the $d_{3}$ rod is shortened and then extended again, which conforms to the principle of rod length variation in the crank slider mechanism. When the shortest position of rod $d_{3}$ is $558.594 \mathrm{~mm}$, it is known from the geometrical relation and the mechanical principle that the mechanism is the dead point of the mechanism and the mechanism can continue to move through the dead point with the help of the $\Psi^{\prime}$ axis; it also verified the correctness of the driving scheme selection.

\section{Conclusion}

In this paper, a new type of 4-UPU parallel mechanism with 4 degrees of freedom is proposed and applied to the automatic pouring equipment for the first time. A mobile pouring robot suitable for complex operating environment is exploited and developed. The main work and conclusions are as follows.

(1) For the first time, the parallel mechanism was proposed to be used in heavy-duty continuous pouring operation. The application of screw theory proves that the 4-UPU mechanism is over constrained mechanism with 4 DOF, can realize space three rotation, and rotate around the $Y$ axis, in which 3 chains of shifting pairs and 1 chains of revolute joints can be used as drive pairs; the modified G-K formula to verify the screw theory to calculate degree of freedom is correct.

(2) The application of screw theory demonstrates the rationality of using 4 th chains as auxiliary chains, and using geometric method, the closed form equations of the kinematic positive and inverse solutions of a new type of 4UPU parallel mechanism are given. Compared with algebraic method, the positive solution method presented in this paper has the advantages of unique solution, high accuracy, and fast calculation speed.

(3) Based on the Bernoulli equation and the model of the volume change of the ladle liquid, an instantaneous section model of pouring liquid flow in ladle was established. The numerical example is validated that the pouring model is correct. Numerical examples are given to verify that the model is correct. The change of each driving pair during the tilting of the fixed position is given, and it is concluded that the rotation of the ladle is the coupling motion between the $d_{3}$ rod and the $\Psi^{\prime}$ shaft. The tilting model built in this paper is very suitable for developing an automatic pouring robot with low cost and no closed loop control and guiding the robot's tilting movement.

\section{Conflicts of Interest}

The authors declare no conflicts of interest related to this paper.

\section{Acknowledgments}

The authors acknowledge the support of the Major Science and Technology Projects in Anhui China: The Development of Heavy Load Casting Robot in Complex Operation Environment (Grant no. 16030901012); thanks are due to $\mathrm{Mr}$. Wang Dong of Jiangsu Fangcun Map Information Technology Co. Ltd. in this paper for providing important technical support.

\section{References}

[1] K. Kozuszek, "Robotic problem solving," Modern Casting, vol. 96, no. 4, pp. 24-28, 2006.

[2] K. Terashima, "Recent automatic pouring and transfer system in foundries," Sokeizai, vol. 39, pp. 1-8, 1998.

[3] P. B. Cervellero and J. J. Mckelvie, "Induction melt shop technologies: part 2," Foundry Management and Technology, vol. 5, pp. 46-50, 1998.

[4] B. F. Benjamin, Of Bridgeton, Indiana. Dumping-Cast, US: $00878115,1908$.

[5] W. R. Smith, Tundish Dumping and Skewing Device for Continuous Casting, US: 03524494, 1970.

[6] L. Wan and Y. Y. Zhao, "Automatic pouring machine with stream inoculation and equal flow pouring," Hot Working Technology, vol. 5, pp. 49-50, 1998.

[7] J. Wu, "Design and application of the hanging and stepping run machine," Research Studies On Foundry Equipment, vol. 1, pp. 31-34, 2002. 
[8] J. Watanabe and K. Yoshida, "Automatic pouring equipment for casting-mel pore system," Industrial Heating, vol. 29, no. 4, pp. 19-27, 1992 (Japanese).

[9] K. Shinohara and H. Morimoto, "Development of automatic pouring equipment," Journal of the Society of Automotive Engineers of Japan, vol. 46, no. 11, pp. 79-85, 1992.

[10] L. Feng, W. Zhang, Z. Gong, G. Lin, and D. Liang, "Developments of delta-like parallel manipulators - a review," Robot, vol. 36, no. 3, pp. 375-384, 2014.

[11] Y. D. Patel and P. M. George, "Parallel manipulators applications-a survey," Modern Mechanical Engineering, vol. 2, no. 3, pp. 57-64, 2012.

[12] M. Ishii and H. Saita, "A new approach to robot teaching method with multimedia tools," IFAC Proceedings Volumes, vol. 28, no. 20, pp. 137-140, 1995.

[13] Y. Noda, K. Terashima, M. Suzuki, and H. Makino, "Weight control of pouring liquid by automatic pouring robot," IFAC Proceedings Volumes, vol. 42, no. 23, pp. 185-190, 2009.

[14] K. Yano and K. Terashima, "Supervisory control of automatic pouring machine," Control Engineering Practice, vol. 18, no. 3, pp. 230-241, 2010.

[15] K. Yano, Y. Sugimoto, Y. Noda, and K. Terashima, "Pouring flow rate control of cylindrical ladle-type automatic pouring robot by applying betterment process," Nippon Kikai Gakkai Ronbunshu C Hen, vol. 16, no. 6, pp. 1750-1757, 2004.

[16] K. Terashima and K. Yano, "Sloshing analysis and suppression control of tilting-type automatic pouring machine," Control Engineering Practice, vol. 9, no. 6, pp. 607-620, 2001.

[17] Z. G. Sun, Y. Y. Liang, and G. Xi, "Numerical study of the flowing sequence of a pouring liquid," Science China Physics, Mechanics and Astronomy, vol. 54, no. 8, pp. 1514-1519, 2011.

[18] Z. Pan, C. Park, and D. Manocha, "Robot motion planning for pouring liquids," in Proceedings of the 26th International Conference on Automated Planning and Scheduling, ICAPS 2016, pp. 518-526, London, UK, June 2016.

[19] Z. Y. Zhu, The Research And The Development of The Fixed Rotary Type of Electric Stove Quota Pouring System, Kunming University of Science and Technology, 2010.

[20] Anhui University Of Science and Technology, Three Degree of Freedom Parallel Hydraulic Support, CN201710-682226.8, China, 2017.

[21] R. Di Gregorio and V. Parenti-Castelli, "Mobility analysis of the 3-UPU parallel mechanism assembled for a pure translational motion," Journal of Mechanical Design, vol. 124, no. 2, pp. 259264, 2002.

[22] J. K. Davidson and K. H. Hunt, Robots and Screw Theory: Applications of Kinematics and Statics to Robotics, Oxford University Press on Demand, London, UK, 2004.

[23] Z. Huang, J. Liu, and D. Zeng, "A general methodology for mobility analysis of mechanisms based on constraint screw theory," Science China Technological Sciences, vol. 52, no. 5, pp. 1337-1347, 2009.

[24] T. L. Yang, H. P. Shen, A. X. Liu, and J. S. Dai, "Review of the formulas for degrees of freedom in the past ten years," Journal of Mechanical Engineering, vol. 51, no. 13, pp. 69-80, 2015.

[25] Y. Noda and K. Terashima, "Modeling and feedforward flow rate control of automatic pouring system with real ladle," Journal of Robotics and Mechatronics, vol. 19, no. 2, pp. 205-211, 2007.

[26] L. E. K. Holappa, "Review of ladle metallurgy," Scandinavian Journal of Metallurgy, vol. 9, no. 6, pp. 261-266, 1980. 


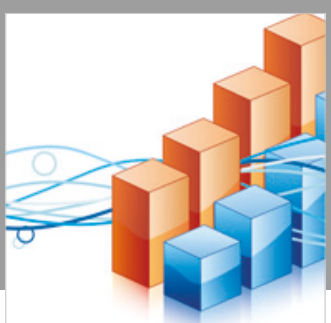

Advances in

Operations Research

\section{-n-m}
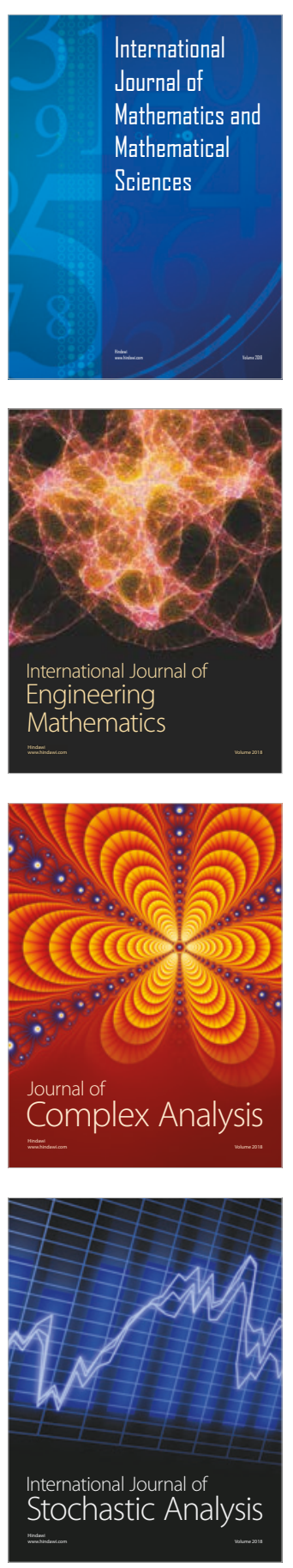
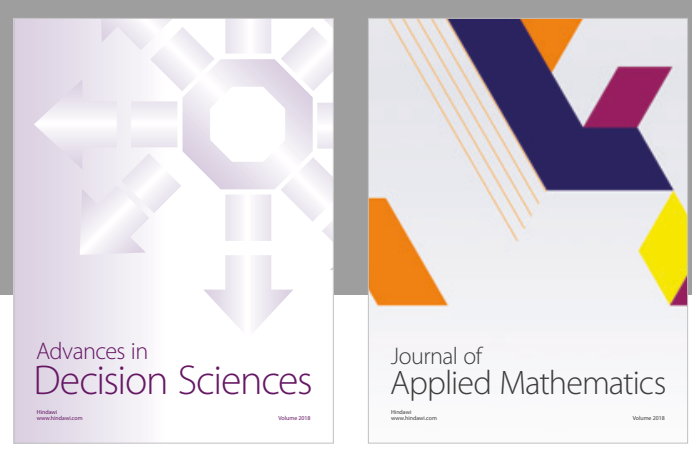

Journal of

Applied Mathematics
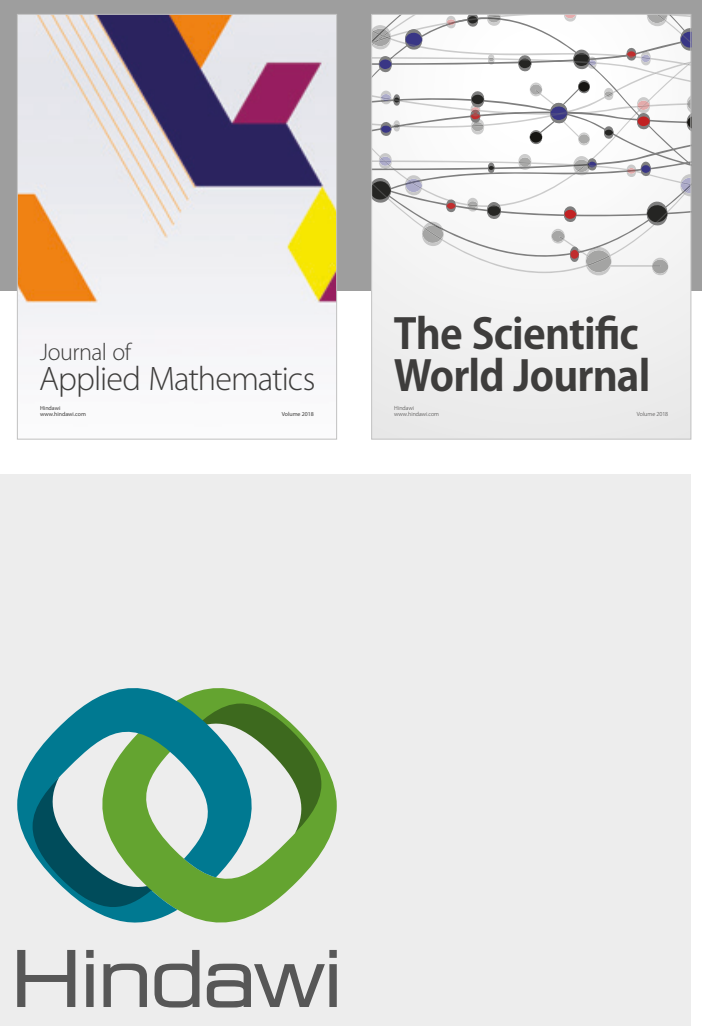

Submit your manuscripts at

www.hindawi.com

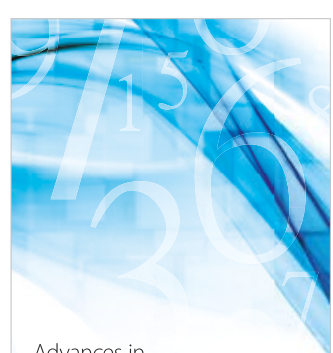

Advances in
Numerical Analysis
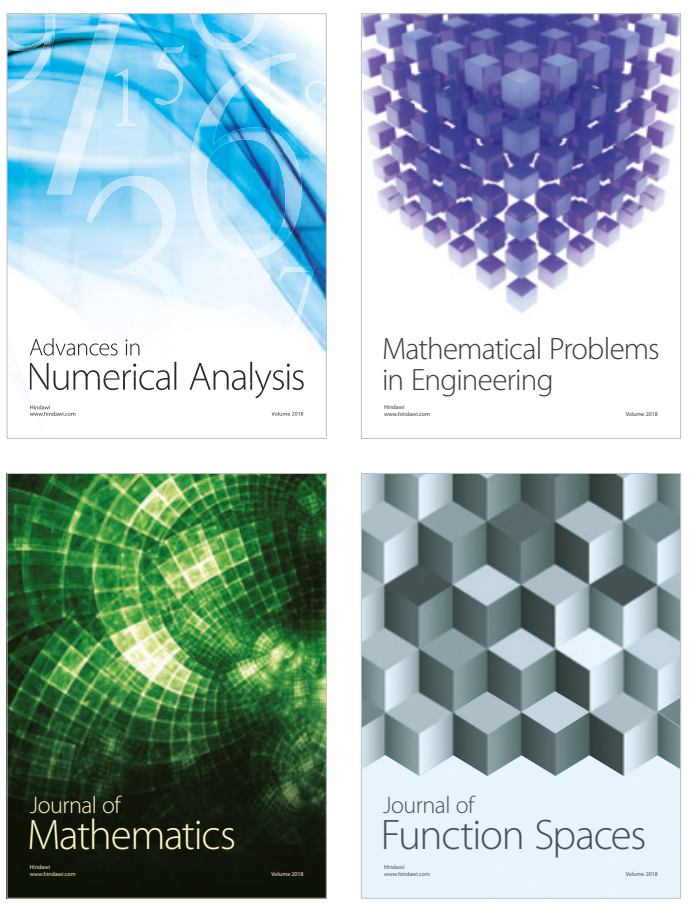

Mathematical Problems in Engineering

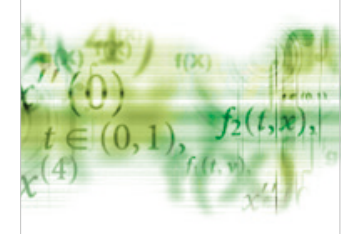

International Journal of

Differential Equations

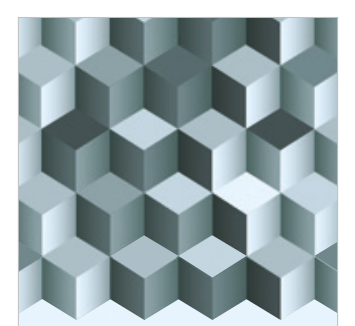

Journal of

Function Spaces

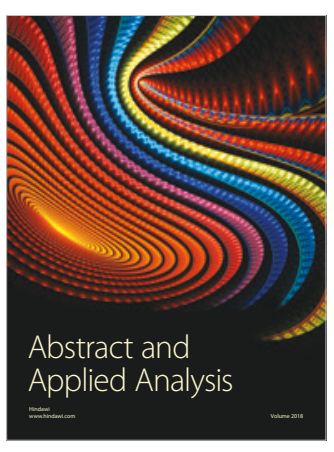

The Scientific

World Journal

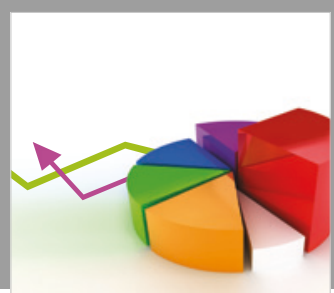

Journal of

Probability and Statistics
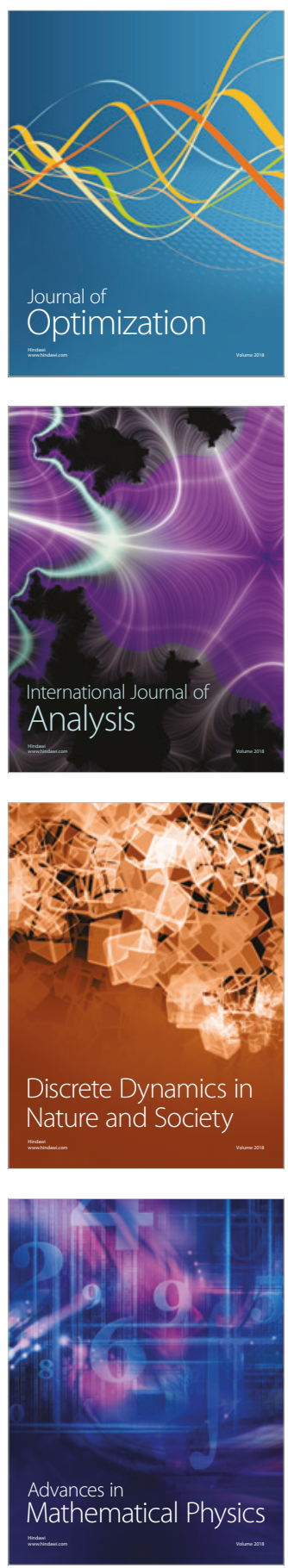